\title{
A Study on the Suitability of Ethernet/IP and EtherCAT for Industrial Time Critical Applications
}

\author{
P. A. Manoj Kumar and B. Sathish Kumar
}

\begin{abstract}
In modern times, industrial communication networks have become an important element for advanced control and automation systems. Various factors including high performance, low installation and maintenance costs, have become major factors while deciding the appropriate protocol standards. However, real-time capabilities are often needed in an increasing number of scenarios.

Ethernet based communication solutions have become increasingly popular in recent times. With the number of installed nodes crossing over one million, it can be safely said that Ethernet technologies are here to stay. Some of the best protocols under this category include Ethernet/IP, PROFINET, Modbus TCP, EtherCAT, Sercos III, and Powerlink. In the following paper we have tried to choose a real time ethernet solution to a specific industrial problem based on their characteristics and suitability in a time critical application.
\end{abstract}

Index Terms—EtherCAT, ethernet/ IP, real time ethernet

\section{The Problem AT HAND}

An electrostatic precipitator is a device used to clean the air flowing out from an industry by removing dust particles using electric charge.

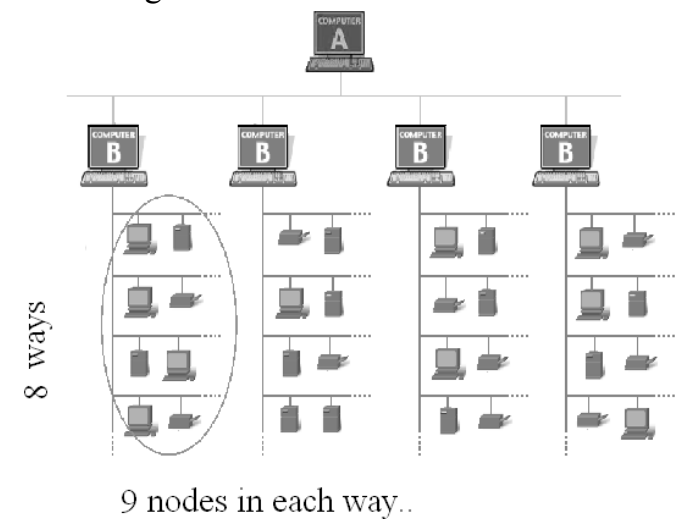

Fig. 1. The Problem at hand - each Electrostatic precipitator consisting of 72 nodes and is connected to the main computer(A) through a secondary computer (B). Maximum permissible time delay while communicating with (A) should be less than 1second.

Consider a typical industrial plant which has 4 electrostatic precipitators that are monitored by a main computer (A) via 4 secondary computers (B). Each precipitator is split into 8 different ways each having 9 different fields. All these are controlled by devices and the instantaneous data are sent to the secondary computers for verification purposes. Thus there are 72 nodes in total in each of the Ethernet cables for

Manuscript received September 15, 2012; revised October 25, 2012.

The authors are with the Indian Institute of Technology, Madras (email : ee10b089@ee.iitm.ac.in,manojpamk@gmail.com, ee10b095@ee.iitm.ac.in, sathishk23@gmail.com ) each electrostatic precipitator. If there is a malfunction sensed by one of the nodes, that information must be sent to the main computer as soon as possible. The time delay in this case should be less than a second.

The problem at hand is, choosing among the present real time Ethernet protocols that fulfill these requirements and finding the best among them that is most suitable with minimum implementing cost.

Out of the many Ethernet protocols available only some fulfill the above requirements. EtherNet/IP, PROFINET, EtherCAT, Sercos III, Modbus TCP, Ethernet Powerlink are the protocols that are suitable for these requirements. In this paper we have discussed the suitability of two of the above mentioned protocols, namely, EtherNet/IP and EtherCAT.

\section{ETHERCAT}

\section{A. Overview}

EtherCAT - Ethernet for Control Automation Technology is a highly flexible Ethernet network protocol. It is being developed at a rapid rate and growing even faster. The operating principle called 'processing on the fly' gives EtherCAT a handful of unique advantages. The principle is that EtherCAT messages are passed before being processed in each node; this process also creates flexibility in topology and incredible synchronization.

In addition to the principle of processing on the fly, EtherCAT benefits from superb infrastructure. Other advantages of EtherCAT include a safety protocol, multiple device profiles and a strong user group. All these ensure EtherCAT is poised for continued growth.

In industrial and building automation systems, typical Ethernet messages for an application layer like Modbus TCP may carry very little data like a valve controller or, in our case value for an electric field. Since only small packets of data are moved and that too only when the Client or Master device requests that data, huge slices of Bandwidth are lost. Similar kinds of bandwidth and payload issues are found in almost all application layers in Ethernet/IP, PROFINET I/O in addition to Modbus TCP. To tackle these low bandwidth utilization issues, a German automation company Beckhoff developed a Fieldbus system called Fast Lightbus. This protocol led to the release of EtherCAT in 2003 by Beckhoff [1].

In 2004, Beckhoff helped to create a new group to promote the EtherCAT protocol. Their efforts led to the EtherCAT Technology Group, or ETG - a forum in which key companies from various industries and leading automation suppliers join forces to support, promote and advance the 
EtherCAT technology. Beckhoff donated the rights to EtherCAT to the ETG.

\section{B. Operating Principle}

The fundamental principle of EtherCAT is "pass-through reading". In this principle, messages are not destined for a single node and consumed by that node. Instead, messages are transmitted to the following node in a string as they are processed. Thus, effectively each string passes through all nodes in the segment. Input data to a node is read as the message is processed and output data is inserted in the message to the next node. A single message is issued by the EtherCAT Master with data for all nodes. As the message is transmitted around the ring and back toward the Master (EtherCAT is implemented on a full duplex architecture, meaning sending and receiving happens on separate lines), each node reads its inputs and adds its outputs to the message. Thus when the message arrives back at the EtherCAT Master every node in the network has received new input data from the Master and returned new output data to the Master.

Overall, the delay as the string passes through each node is a few nanoseconds [2].Without the deficiency of small payloads or messages targeted to specific nodes, an EtherCAT network can achieve maximum bandwidth utilization. An EtherCAT network can be compared to a railway where each station can off-load and re-load train cars while the train moves through the station.

\section{The EtherCAT frame}

The EtherCAT frame replaces the Ethernet Data portion of an Ethernet Packet, often IP data

All parts of the IP data fit simply into the EtherCAT frame, and the frame fits simply into an Ethernet frame. The Ethernet is the transmission media that allows EtherCAT to operate. The EtherCAT frame simply replaces the IP frame of a standard Ethernet message. Thus, the Ethernet frame does not need modification, again contributing to flexibility for EtherCAT.

The EtherCAT frame can be easily studied using the analogy of a train. The frame passes through all the nodes without pausing. Each node can thus access all the blocks in the frame. It may be noted that each sub - telegram itself may be of variable length. As the frame passes through a node, it extracts the commands (data) that has been assigned to it and inserts back other I/O data. A particular node can extract or insert single bits or entire block of data from the frame as it passes though. This technique is known as "Processing on the Fly". The Process data size per slave (node) is almost unlimited, varying from 1 bit to $60 \mathrm{~KB}$ [3].

\section{Synchronization}

EtherCAT Node measures time difference between leaving and returning frame. There are Distributed Clocks which do precise synchronization by exact adjustment. As specified in the IEEE 1588 Precision Time Protocol Standard, Synchronization has gained importance in the Industrial Networking industry. It is another advantage of EtherCAT systems. Since EtherCAT uses precisely adjusted distributed clocks, the communication cycle itself does not have to be absolutely equidistant, that is, a small jitter is allowed.

Each node includes timestamps in the EtherCAT frame twice. The slave node adds a timestamp when a message is received as it is sent through the network and adds another timestamp when the frame returns back to the node. The master slave receives the frame with two timestamps per slave. The master can calculate the delay for each node with this time information. This process is repeated by the master for every frame it sends. The master has incredibly accurate data as the network operates. The ring topology creates an incredibly accurate clock mechanism.

Measurements showed a synchronization accuracy of (approx.) 20ns with 300 distributed nodes and 120m cable length [4]. Since the maximum jitter depends on various network characteristics, its value is conservatively given to be much less than $1 \mu \mathrm{s}$.

\section{E. Performance Characteristics}

The EtherCAT performance is measured using the minimum achievable cycle time as a function of number of slaves/nodes. The various parameters which affect the cycle time are:

Forwarding time of the packet at the master $-T_{m}^{f}$

Maximum delay of the Physical Layer $-T_{p}$

Propagation delay along the cables $-T_{c}{ }^{p}$

Forwarding time of the packet inside the slave $-T_{s}^{f}$

Number of slaves $-n$

The total delay per EtherCAT slave in a line topology is

$$
T_{s}^{\text {tot }}=T_{p}+T_{c}^{p}+T_{s}^{f}
$$

Realistically the total slave forwarding delay and the cable propagation delay associated with each slave can be set to $1 \mu \mathrm{s}$ [5]. Thus the minimum achievable cycle time can be obtained as

$$
T_{\min }^{\text {line }}=T_{m}^{f}+T_{p}+n \times 1 \mu s
$$

Suppose $T_{1538}$ is the forwarding time of a maximum sized packet, the corresponding expression for multiple packets becomes more complex and is given by

$$
T_{\text {line }}^{\min }=(k-1) \times T_{1538}+T_{\text {rest }}+T_{p}+n \times 1 \mu s
$$

The cycle time for a payload of 100 bytes per node has been plotted as a function of the number of nodes in Fig. 2.

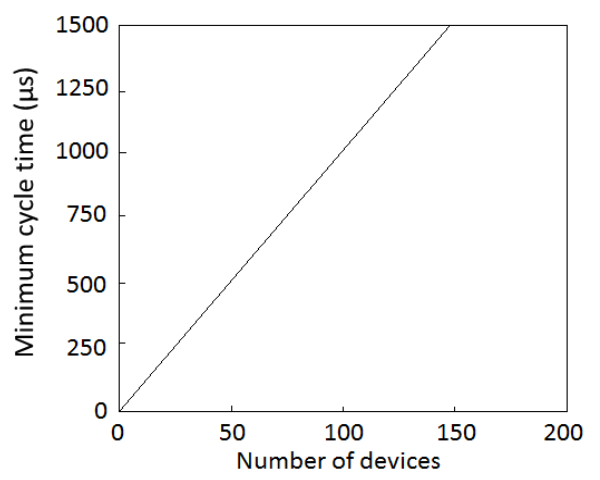

Fig. 2. EtherCAT cycle time as a function of number of nodes, with 100 bytes payload per device 
The above analysis of EtherCAT shows that cycle times as small as hundreds of microseconds can be achieved for the 72 nodes in our problem. A similar study of Ethernet/IP on the basis of a different parameter will now be presented.

\section{ETHERNET/IP}

\section{A. Overview}

Ethernet/IP is a communications protocol developed by Rockwell Automation in 2001. It is currently maintained by ODVA (open Device Net Vendors Association); Control Net International and the IEA (Industrial Ethernet Association) [2]. Ethernet/IP employs CIP (Common Industrial Protocol) in its Application, Sessions and Presentation layers, TCP/IP or UDP/IP in its Networking and Transport Layers and is built on the existing IEEE 802.3 Physical/Data Link Layers. This structure of Ethernet/IP provides a significant compatibility with CIP standards such as Device Net and Control Net and ease of implementation in existing ethernet based systems. It supports real-time I/O messaging, explicit message exchange or both.

When strict guidelines are used, EIP offers a real time solution, but it is not deterministic. In the application layer it uses CIP [6]. CIP is a well suited protocol for distributed systems such as object orientation, Electronic Data Sheets and device profiles. EIP with only CIP does not give a real time protocol. To get a real time protocol CIPSync should be employed. EIP uses both TDP and UDP for communication. TCP is used when a connection oriented exchange is preferred. For real time traffic EIP uses UDP. Network collisions are avoided by switches. EIP is generally implemented in star topology.

\section{B. Operating Principle}

Ethernet/IP belongs to the class of Industrial Ethernet protocols which make use of standard ethernet hardware and standard TCP/IP and UDP/IP stacks for communication.

Under CIP, communication between nodes takes place using the producer - consumer model. Here, a message intended for particular node(s) is transmitted to all the nodes. However the message is filtered once it is received by the nodes and hence only reaches the node(s) it was intended for. This is illustrated in Fig. 3. Although this is very effective in slave to slave communication; it requires broadcasting and filtering in each node.

Moreover, during broadcasting, the switches do not have any particular role but to forward all the incoming packets, thus behaving rather as hubs.

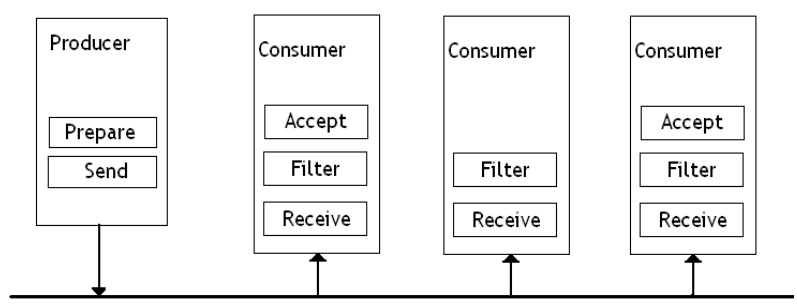

Fig. 3. The producer consumer model

\section{Ethernet/IP Frame Structure}

Ethernet/IP frame is similar to an Ethernet frame with some additional features (Fig. 4) It has a 7 byte preamble which is used by the receiver to allow it to establish bit synchronization. It is followed by a start frame delimiter (1 byte) which indicates the start of the frame.

The next 12 bytes are the MAC addresses of the destination and the source - first 6 bytes for the destination address and the next 6 for the source address. The next two bytes specify the EtherType which specifies the protocol that is in use in that network. From there on the payload of the frame follows. Its length can be anything between 46 to 1500 bytes. Padding is optional. It is only necessary if the data packet is less than 46 octets. The frame ends with a 4 byte long Frame Check Sequence (FCS) which guarantees the integrity of the received data.

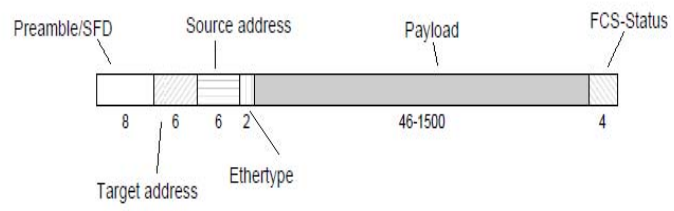

Fig . 4. A typical ethernet frame - The ethertype specifies the protocol in use

\section{Synchronization}

Synchronization in Ethernet/IP is achieved through the CIP Sync - making use of IEEE 1588 Standard and Distributed Clock Protocol. CIPSync can achieve a synchronization accuracy of better than 500ns, without considering jitters caused due to protocol stacks [2].

IEEE 1588 is a method for precision time synchronization tailored to requirements of distributed measurement and control systems. However, in practice it has been observed that stack processing times limit the accuracy in case of pure software implementations, and hence to achieve better results, hardware time stamping is required. This functionality can be implemented in MAC (Media Access Controls), the Physical Layers or integrated solutions.

\section{E. Performance Characteristics}

The performance of Ethernet/IP depends on the number of CIP connections. It also depends on the topology of the network to a smaller extent. Each device can have any number of CIP connections. In the following, we have assessed the performance of Ethernet/IP by listing out the Requested Packet Interval (RPI) times [4]. The RPI specifies the period at which data updates over a connection. For example, an input module sends data to a controller at the RPI.

The minimum RPI (Requested Packet Interval) is given by

min_rpi $=\frac{\text { number of connections } \times 2}{\text { number of frames per sec ond }}$

It must be noted that the above values do not take into account switch and remote stack delays into account. Rockwell Automation quantifies the switch delay at $0.1 \mathrm{~ms}$ per switch.

Consider the case of 64 CIP connections. We have approximated the RPI for our network with 72 connections using the data from the last row. The above table suggests that a frame will take at least $25.6 \mathrm{~ms}$ to travel from one 
module to another when the media can accommodate 5000 frames per second. Thus with 72 nodes the minimum RPI with all nodes trying to communicate is $1.84 \mathrm{~s}$ (approx). Obviously this is much more than our permissible delay of 1 second. However considering 25000 frames per second, the same delay reduces to $368.64 \mathrm{~ms}$, which is acceptable keeping in mind switch and stack delays which have not been considered in the above table.

\begin{tabular}{|c|c|c|c|}
\hline $\begin{array}{l}\text { No. of } \\
\text { Connections }\end{array}$ & $\begin{array}{l}\text { Standard } \\
\text { Scanners } \\
(5000 \\
\text { Frames/sec })\end{array}$ & $\begin{array}{l}\text { High } \\
\text { Performance } \\
\text { Scanners } \\
(10000 \\
\text { Frames/sec) }\end{array}$ & $\begin{array}{l}\text { Ultra high } \\
\text { performance } \\
\text { scanners }(25000 \\
\text { Frames/sec) }\end{array}$ \\
\hline 4 & 1.6 & 0.8 & 0.32 \\
\hline 8 & 3.2 & 1.6 & 0.64 \\
\hline 16 & 6.4 & 3.2 & 1.28 \\
\hline 32 & 12.8 & 6.4 & 2.56 \\
\hline 64 & 25.6 & 12.8 & 5.12 \\
\hline
\end{tabular}

Thus the performance constraint of data transfer within a second is possible in the case of Ethernet/IP only with the use of Ultra high scanners, which can provide a capacity of 25000 frames/second in the media.

\section{CONCLUSION}

In this paper we have taken the case of a specific industrial communication problem and tried to present a real time ethernet networking solution for the same. A detailed analysis of two of the fast emerging solutions - EtherCAT and Ethernet/IP, has been undertaken on the basis of their frame structures, synchronization and performance characteristics. Since the working principles of the two protocols are quite dissimilar, a separate discussion for each has been presented.

EtherCAT is expected to perform well within the constraint of data transfer within one second. Moreover it is relatively simpler to implement and is available as open source code. Ethernet/IP on the other hand, has a much larger footprint with installed capacity in over a million nodes, but requires special hardware (Ultra high performance scanners) to achieve data transfer rate of less than a second.

\section{ACKNOWLEDGMENT}

We would like to extend our acknowledgment to the Department of Electrical Engineering, IIT Madras for providing a platform to choose and undertake the above study. We would also like to thank the Management - Central Library, IIT Madras for providing us access to Scientific Journals and Papers which helped us immensely in our work.

\section{REFERENCES}

[1] Open Real-Time Ethernet Network. [Online]. Available: http://www.rtaautomation.com/ethercat/

[2] P. Doyle, "Introduction to Real time Ethernet II,"- The Extension, vol. 5, no. 4, July-August 2004

[3] EtherCAT-The Ethernet Fieldbus. [Online]. Available: http://www.ethercat.org/pdf/english/EtherCAT_Introduction_0905.pd $\mathrm{f}$

[4] ETG Industrial Ethernet Seminar Series, EtherCAT Technology Group, 2011

[5] G. Prytz, "A Performance analysis of EtherCAT and Profinet IRT," in Proc. of $13^{\text {th }}$ IEEE International Conference on Emerging technologies and Factory Automation Hamburg, Germany, 2008, pp.408-415

[6] Ethernet/IP $P^{T M}$ - CIP on Ethernet Technology, The CIP Advantage Technology Overview Series, Open Device Net Vendors Association, (C) 2006-2008 ODVA, Inc.

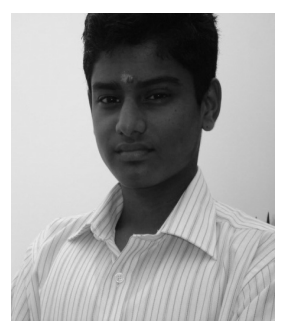

P. A. Manoj Kumar is a student of the Department of Electrical Engineering, Indian Institute of Technology, Madras. He is a Dual Degree student, pursuing Bachelors in Electrical Engineering and Masters in Communication Engineering. He has developed a' Crosswords' program in $\mathrm{C}++$. He has received the Srinivasa Ramanujam Academy Award of Maths Talent and $\mathrm{N}$ Subramaniam Memorial Award for securing highest scores in Maths and English in AISSCE 2010 respectively. He has also done a project on 'Analysis of wave forms using VHDL Coding'.

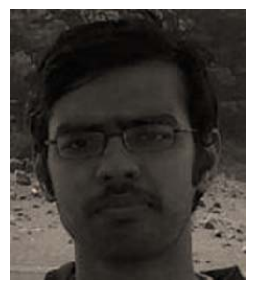

B. Sathish Kumar is a student of the Department of Electrical Engineering, Indian Institute of Technology, Madras. He is a Dual Degree student, pursuing Bachelors in Electrical Engineering and Masters in Communication Engineering. He has secured the third highest aggregate marks in AISSCE 2010 in his district. 